stroke alerts alongside physicians and facilitate timely triage. Imaging triage involves CT angiography and CT perfusion at $57 \%$ of institutions, CT angiography without CT perfusion at $30 \%$, and some use of MRI at $10 \%$. During the team activation step, the neurointerventionalist was contacted prior to completion of non-invasive imaging at $86 \%$ of institutions, and thus before triage was a decision to treat could be made. Likewise, the remaining neurointerventional team members were called in prior to making a decision to treat at 59\% of institutions. Team members were contacted directly by the neurointerventionalist at $63 \%$ of institutions, and by a hospital operator at $23 \%$. Once activated, the mean required arrival time for nurse and technologist staff was 30 minutes. During the transport workflow step, patients were permitted to be transported to the neurointerventional suite before team arrival at $43 \%$ of institutions. Emergency department staff were involved in transport at $87 \%$ of facilities, while the neurointerventional team was involved at only $20 \%$. For the case preparation step, procedural trays were set up in advance of team arrival at only $13 \%$ of institutions. Thrombectomy devices were stored in a centralized, easy-to-find location at 54\% of centers. A power injector for angiographic runs was consistently used at $43 \%$, but no institution left the injector loaded in anticipation of cases. Anesthesiology routinely participated in thrombectomies at $67 \%$ of institutions, though general anesthesia was only used consistently in only $21 \%$.

Conclusion Workflow processes related to triage, team activation, transport, and case preparation prior to mechanical thrombectomy vary widely between institutions. These differences may reflect institution-specific factors or incomplete awareness of best practices. Broader dissemination of best practices and successful workflows may allow institutions to develop more efficient systems of care than would otherwise be possible.

Disclosures A. Kansagra: None. G. Meyers: None. M. Kruzich: None. D. Cross: None. C. Moran: 2; C; Medtronic Neurovascular. 3; C; Medtronic Neurovascular. C. Derdeyn: None.

\section{0-011 SYMPTOMATIC INTRACRANIAL HEMORRHAGE AFTER REPERFUSION THERAPY - IMPACT OF DEFINITION ON ITS FREQUENCY}

${ }^{1} \mathbf{R}$ von Kummer, ${ }^{2} \mathrm{D}$ Frei, ${ }^{3} \mathrm{~A}$ Yoo, ${ }^{4} \mathrm{O}$ Zaidat, ${ }^{5} \mathrm{P}$ Khatri, ${ }^{6} \mathrm{R}$ Gupta, ${ }^{7} \mathrm{D}$ Lopes, ${ }^{8} \mathrm{H}$ Shownkeen, ${ }^{9} \mathrm{D}$ Meyer, ${ }^{9} \mathrm{H}$ Buell, ${ }^{9} \mathrm{~V}$ Bach, ${ }^{9} \mathrm{~S}$ Kuo, ${ }^{9} \mathrm{~A}$ Bose, ${ }^{9} \mathrm{~S}$ Sit, ${ }^{10} \mathrm{~J}$ Mocco. ${ }^{1}$ Universitätsklinikum Carl Gustav Carus, Dresden, Germany; ${ }^{2}$ Swedish Medical Center, Englewood, CO; ${ }^{3}$ Texas Stroke Institute, Plano, TX; ${ }^{4}$ St Vincent Mercy Hospital, Toledo, OH; ${ }^{5}$ University of Cincinnati, Cincinnati, OH; ${ }^{6}$ WellStar Health System, Marietta, GA; ${ }^{7}$ Rush University Medical Center, Chicago, IL; ${ }^{8}$ Central DuPage Hospital, Winfield, IL; ${ }^{9}$ Penumbra, Inc., Alameda, CA; ${ }^{10}$ Mount Sinai Health System, New York, NY

\subsection{6/neurintsurg-2016-012589.11}

Introduction There is currently no standardized criterion for a clinically defined symptomatic intracranial hemorrhages (sICH), particularly secondary to endovascular therapy of ischemic stroke. Recent randomized controlled trials evaluating the efficacy and safety of intra-arterial therapy have published results with varying complication rates based upon the sICH definition employed. To further elucidate the variability in sICH outcome, the present study subjects the THERAPY cohort to definitions of sICH from similar trials.

Materials and methods The multicenter THERAPY trial enrolled a total of 108 patients randomized to either IV-tPA monotherapy or a combined thrombolytic and intra-arterial therapy. Angiography was performed at presentation and at 24 hours from intervention. To meet the present study criterion, patients included in the study must have the relevant data document for evaluation of sICH presence, defined in the THERAPY trial as $24 \mathrm{~h} \mathrm{CT} \mathrm{evidence}$ of an ECASS-defined intracranial hemorrhage and a worsening of $\geq 4$ on the National Institute of Health Stroke Scale and compared to outcomes when subjecting the same cohort to definitions from related trials.

Results In total, 105 patients from the THERAPY trial met the criteria for analysis. Under the study definition (Definition A), the rate of sICH was observed to be $9.5 \%$ (10/105) for both arms of the THERAPY trial, with 9.3\% (4/43) of patients documented for the combined therapy arm. More narrow definitions for sICH, such as those employed by

Abstract 0-011 Table 1

\begin{tabular}{|c|c|c|c|c|c|c|c|c|}
\hline \multirow[t]{2}{*}{$\begin{array}{c}\text { Stroke } \\
\text { Trials }\end{array}$} & \multicolumn{2}{|c|}{$\begin{array}{l}\text { Definition A: } \\
\text { 24hr CT evidence of an } \\
\text { ECASS defined ICH and a } \\
\text { worsening in NIHSS score of } \\
\geq 4 \text { from baseline }\end{array}$} & \multicolumn{2}{|c|}{$\begin{array}{l}\text { Definition B: } \\
\text { Any PH1, PH2, RIH, SAH, or } \\
\text { IVH associated with a } \geq 4 \\
\text { points worsening on the } \\
\text { NIHSS within } 24 \mathrm{hr}\end{array}$} & \multicolumn{2}{|c|}{$\begin{array}{c}\text { Definition C: } \\
\text { PH2 within } 36 \mathrm{hr} \text { of } \\
\text { treatment combined with } \geq 4 \\
\text { point increase in NIHSS from } \\
\text { baseline, or the lowest } \\
\text { NIHSS value between } \\
\text { baseline and } 24 \mathrm{hr}\end{array}$} & \multicolumn{2}{|c|}{$\begin{array}{c}\text { Definition D: } \\
\text { New intracranial } \\
\text { hemorrhage (ICH, SAH, IVH, } \\
\text { SDH) associated with clinica } \\
\text { evidence of neurological } \\
\text { worsening associated with } \\
\text { NIHSS score of } \geq 2 \text { points } \\
\text { different from baseline }\end{array}$} \\
\hline & Control & Intervention & Control & Intervention & Control & Intervention & Control & Intervention \\
\hline ESCAPE & - & - & - & - & - & - & $2.7 \%$ & $3.6 \%$ \\
\hline EXTEND IA & - & - & - & - & $6 \%$ & $0 \%$ & - & - \\
\hline REVASCAT & $1.9 \%$ & $4.9 \%$ & - & - & $1.9 \%$ & $1.9 \%$ & - & - \\
\hline $\begin{array}{l}\text { SWIFT } \\
\text { PRIME }\end{array}$ & - & - & $3.1 \%$ & $0.0 \%$ & - & - & - & - \\
\hline MR CLEAN & $6.4 \%$ & $7.7 \%$ & - & - & - & - & - & - \\
\hline THERAPY & $9.7 \%$ & $9.3 \%$ & $4.8 \%$ & $2.3 \%$ & $4.8 \%$ & $0.0 \%$ & *N/A & ${ }^{*} \mathrm{~N} / \mathrm{A}$ \\
\hline
\end{tabular}

* Adjudication in progress

Note: $\mathrm{ICH}=$ Intracranial Hemorrhage, $\mathrm{PH}=$ Parenchymal Hemorrhage, RIH = Remote Intracranial Hemorrhage, SAH = Subarachnoid

Hemorrhage, IVH = Intraventricular Hemorrhage, SDH = Subdural Hemorrhage 
REVASCAT and SWIFT PRIME, demonstrated a reduction in the rate for sICH of the THERAPY cohort to as low as $0.0 \%$ for the intervention arm (Table 1).

Conclusion Under the trial definition, any apparent extravascular blood in the brain or cranium with an increase of 4 or more on the NIHSS, associated or not, is considered a sICH; as a result, the inclusion of hemorrhagic infarctions (HI1 and HI2) in this definition resulted in an apparent relative increase in sICH rates. Subjecting the THERAPY cohort to other definitions yielded notable variance in complication rates. As there is no current standardization for the quantification of sICH, further analysis and data are needed to identify and standardize a succinct and relevant definition.

Disclosures R. von Kummer: 2; C; Penumbra, Inc. D. Frei: 3; C; Penumbra, Inc. A. Yoo: 1; C; National Institute of Health, Penumbra, Inc., Remedy Pharmaceuticals. O. Zaidat: 6; C; Penumbra, Inc. P. Khatri: 1; C; Penumbra, Inc. R. Gupta: 6; C; Penumbra, Inc. D. Lopes: 6; C; Penumbra, Inc. H. Shownkeen: None. D. Meyer: 5; C; Penumbra, Inc. H. Buell: 5; C; Penumbra, Inc. V. Bach: 5; C; Penumbra, Inc. S. Kuo: 5; C; Penumbra, Inc. A. Bose: 4; C; Penumbra, Inc. 5; C; Penumbra, Inc. S. Sit: 4; C; Penumbra, Inc. 5; C; Penumbra, Inc. J. Mocco: 1; C; Penumbra, Inc.

\section{0-012 EMERGENT ENDOVASCULAR MANAGEMENT OF LONG- SEGMENT CAROTID ARTERY DISSECTIONS IN ACUTE ISCHEMIC STROKE INTERVENTION WITH MULTIPLE TANDEM STENTS}

${ }^{1} \mathrm{~S}$ Ansari, ${ }^{2} \mathrm{~A}$ Kuhn, ${ }^{3} \mathrm{~A}$ Honarmand, ${ }^{4} \mathrm{~S}$ Hou, ${ }^{5} \mathrm{M}$ Khan, ${ }^{2} \mathrm{~J}$ Chueh, ${ }^{2} \mathrm{I}$ van der Bom, ${ }^{1} \mathrm{M}$ Hurley, ${ }^{1} \mathrm{~A}$ Shaibani, ${ }^{2} \mathrm{M}$ Gounis, ${ }^{1} \mathrm{M}$ Potts, ${ }^{1} \mathrm{~B}$ Jahromi, ${ }^{2} \mathrm{~A}$ Wakhloo, ${ }^{2} \mathrm{~A}$ Puri. ${ }^{1}$ Radiology and Neurological Surgery, Northwestern University Feinberg School of Medicine, Chicago, IL; ${ }^{2}$ Radiology, University of Massachusetts, Worcester, MA; ${ }^{3}$ Radiology, Northwestern University Feinberg School of Medicine, Chicago, IL; ${ }^{4}$ Stroke and Neurovascular center of Central California, Santa Barbara, CA; ${ }^{5}$ Neurology, University of Massachusetts, Worcester, MA

\subsection{6/neurintsurg-2016-012589.12}

Background and purpose Cervical dissections are a significant cause of acute ischemic stroke, especially in young and middle aged adults. Although medical management of cervical dissections is standard treatment, emergent endovascular treatment may become necessary in the presence of acute intracranial large vessel occlusions, flow limiting and long segment lesions with impending occlusion, and/or ischemia at risk for cerebral infarction. We report our experience with the endovascular reconstruction of long segment carotid dissections using multiple tandem stents in acute ischemic stroke intervention.

Materials and methods We retrospectively studied patients with carotid artery dissections requiring stent reconstruction at our institutions between January 2011 and January 2015, presenting with acute ( $<12$ hours), severe ischemic stroke symptoms (NIHSS $>4$ ), and requiring carotid reconstruction with multiple tandem stents ( $\geq 3$ stents). We analyzed patients' demographics, vascular risk factors, presentations, imaging and angiographic findings, technical efficacy and safety, and clinical outcomes.

Results We identified 15 patients presenting with acute ischemic stroke that underwent endovascular stent reconstruction of carotid dissections for vessel and/or ischemic tissue salvage. Mean patient age was 51.5 years with a mean presenting NIHSS of 15 and discharge NIHSS of 6. All carotid dissections presented with $>70 \%$ stenosis and severe flow limitation, nearly all 14/15 involving the distal cervical segment of the ICA with a minimum length of $3.5 \mathrm{~cm}$. Technical success of carotid stent reconstruction was achieved in all patients $(100 \%)$ with no significant residual stenosis or flow limitation. Nine patients $(60 \%)$ harbored simultaneous intracranial occlusions, and 6 patients (40\%) required IA thrombolysis/thrombectomy achieving TICI 2 b-3 reperfusion in all 15 patients. There were no symptomatic intracranial hemorrhages. Procedural complications were limited to distal thromboemboli and multifocal infarcts in a single patient due to suspected in-stent thrombus, resulting in 7\% procedural morbidity and $0 \%$ mortality. Follow-up 3-6 month angiographic and ultrasound imaging evaluations confirmed normalization of carotid artery caliber and stent patency in 12/14 patients, with 2 cases of only mild persistent vessel irregularity and $<20 \%$ in-stent stenosis. On clinical follow-up, 9/15 (60\%) of patients achieved mRS $\leq 2$ at 90 days, with no interval recurrent TIAs or strokes.

Conclusion Tandem stent reconstruction for the treatment of long segment and flow limiting carotid dissections is technically safe and effective with favorable clinical outcomes in acute ischemic stroke intervention, allowing for successful thrombectomy, vessel salvage, restoration of cerebral perfusion, and prevention of recurrent thromboembolic stroke.

Disclosures S. Ansari: None. A. Kuhn: None. A. Honarmand: None. S. Hou: None. M. Khan: None. J. Chueh: None. I. van der Bom: None. M. Hurley: None. A. Shaibani: None. M. Gounis: None. M. Potts: None. B. Jahromi: None. A. Wakhloo: None. A. Puri: None.

\section{0-013 HYPERTENSION DECREASES THE NUMBER AND DURATION OF COLLATERAL OPENINGS DURING MIDDLE CEREBRAL ARTERY OCCLUSION (MCAO) AND IMPAIRS REPERFUSION}

M Cipolla, J Sweet, K Chan. Neurological Sciences, University of Vermont, Burlington, VT

\subsection{6/neurintsurg-2016-012589.13}

Introduction Hypertension is associated with large ischemic cores and little penumbral tissue that is thought to contribute to poor outcome from ischemic stroke. Pial collaterals (leptomeningeal anastomoses; LMA) are thought to perfuse the penumbra during MCAO by promoting retrograde flow from the anterior cerebral artery (ACA) to the MCA territory. We recently demonstrated that pial collaterals are vasoconstricted in spontaneously hypertensive rats (SHR) that likely contributes to poor collateral flow and increased infarction. Here, we developed methodology to measure collateral openings in SHR $(\mathrm{n}=6)$ compared to normotensive Wistar $(\mathrm{n}=6)$ rats during MCAO. We hypothesized that SHR have fewer collateral openings and/or shorter duration openings during MCAO, and impaired reperfusion.

Materials and methods Dual laser Doppler probes were used to simultaneously measure changes in relative cerebral blood flow (rCBF) in the MCA (probe 1: Bregma -2, lateral +4) and ACA collateral (probe 2: Bregma +2 , lateral +3 ) vascular territories during 2 hours of MCAO by filament occlusion (Figure 1). Collateral openings were defined as increased collateral flow that did not correspond to changes in blood pressure. The number and duration of collateral openings were quantified off rCBF tracings blinded to group. Animals were 